\title{
The Impact of the COVID-19 Pandemic and Lockdown on Prevalence of and Risk Factors for Neck Pain Among College Students: a Cross- sectional Study
}

Amira Daher ( $\square$ amiradaher99@gmail.com )

Zefat Academic College

Ofra Halperin

Department of Nursing, Max Stern Academic College of Emek Yezreel

\section{Research Article}

Keywords: Neck pain (NP), COVID-19 pandemic, lockdown, risk factors, college students, cross-sectional study

Posted Date: July 22nd, 2021

DOl: https://doi.org/10.21203/rs.3.rs-701878/v1

License: (c) (1) This work is licensed under a Creative Commons Attribution 4.0 International License. Read Full License 


\section{Abstract}

Background: Neck pain (NP), one of the most reported musculoskeletal disorders, is a major cause of illness and absence from university lessons among students. The COVID-19 pandemic affected higher education institutions worldwide. Academic studies shifted to online-learning, changing students' habits and lifestyle. Data demonstrated that the pandemic and consequent lockdowns can affect people's physical and mental well-being. Thus, the aim of this study is to evaluate the impact of COVID-19 lockdowns on the prevalence of and risk factors of NP among college students compared to the preepidemic period.

Methods: A cross-sectional study was conducted in the form of an online questionnaire survey that included sociodemographic data, Visual Analogue Scale (VAS), Neck Disability Index (NDI), and Perceived Stress Scale (PSS). NP was reported at four time points-lifetime, last year, last six months, and currenton a scale ranging from never/seldom to almost every day. Logistic regression models were calculated for all study variables and compared to the (NDI) cut-off score of 15 .

Results: A total of 295 college students were recruited. Among them, 35.6\% reported at least moderate neck-related disability (NDI $\geq 15)$ and significantly more NP than before the lockdown. NP increased gradually and significantly (on a scale 1-4), from a lifetime mean of 1.80 to a mean of 2.57 last year to 2.73 during the past six months to the current mean of $3.07(p<.001)$. Participants' scores on the PSS were moderate, with $59.3 \%$ reporting a high level of study-related stress. Logistic regression models revealed that sitting more than three hours a day, high study-related stress and higher PSS and VAS scores were associated with higher risk of at least a moderate level of neck-related disability $(R 2=.513, p<$ $.001)$.

Conclusions: The transition from on-campus studies to online learning had negative effects on students. It significantly increased study-related stress and the development of NP. Identifying risk factors at an early stage may prevent NP from becoming chronic and affecting students' future careers, thereby improving students' quality of life.

\section{Background}

Over the past decades, neck pain (NP) has increasingly become a health problem, exerting a considerable socioeconomic impact on individuals, families and communities [1, 2, 3, 4]. Most patients with disabling NP show improvement in pain intensity over 1 year. However, a quarter present of them showed no or minimal improvement in pain intensity [5]. NP was found to be associated with psychosocial and mental stress [6]. Furthermore, the increased severity of NP places a huge burden on the healthcare system [4].

NP is one of the most reported musculoskeletal disorders and is a major cause of illness, reduced educational attainment and absence from university lessons, thus affecting students' future careers [1]. In addition to the factors that predispose people in general to experience NP; students spend long hours reading, writing and using computers or tablets, making them a high-risk group for developing NP [4]. 
In March 2020, the World Health Organization (WHO) determined that COVID-19 can be characterized as a pandemic [7]. WHO and public health authorities around the world acted to contain the COVID-19 outbreak. Israel went into its first lockdown on 15 March 2020 to prevent the spread of COVID-19 infections. The third lockdown in Israel began on January 8, 2021 and lasted until February 7, 2021. Beginning with the first lockdown, academic studies in Israel were required to shift to online learning. The transition from on-campus studies to online studies facing screens has significantly changed students' habits and lifestyle $[3,8,9]$.

The pandemic and the resultant lockdowns restricting people to their homes can affect both their physical and their mental well-being. Research found that the pandemic and consequent lockdowns were associated with depressive symptomatology among both office workers and students $[3,7]$. Home confinement, physical inactivity, lack of academic schedule and stress among students were also found to lead to hormonal imbalance symptoms [3].

Lockdown is an effective way to prevent the transmission of the COVID-19 virus [10]. Yet it may also have negative effects on the musculoskeletal system [3]. Although many cross-sectional and epidemiological studies have examined the prevalence of lower back pain in students [11-14], far fewer studies have focused on NP and its association with factors that can potentially affect students, such as excessive exposure to screens and maladaptive ergonomic environment [12, 14].

A significant positive correlation has been demonstrated between the duration of mobile phone use and the duration and severity of NP [1]. Indeed, musculoskeletal pain in students manifested most often in the neck [11]. Even if NP is a common problem in this group, knowledge is lacking about the development of NP during the coronavirus pandemic.

The COVID-19 pandemic and its associated closures have had a negative impact on the general population. Staying at home leads to social isolation, and consequently to loneliness, anxiety and depression $[15,16]$. In addition, a more sedentary lifestyle, excessive exposure to screens and abnormal sleeping habits can all result in higher body mass index -(BMI) $[3,15]$. During home confinement, undergraduate students were found to experience chronic stress, headaches and lower back pain [17]. Thus, we hypothesize that the incidence of NP among students will be greater during the COVID-19 pandemic and the consequent quarantines than in the period prior to the pandemic.

The main purpose of this study was to evaluate the prevalence of NP among college students during the pandemic, particularly during the third lockdown in Israel, and to compare this rate to the pre-epidemic period, at three time points: lifetime, last year, last six-month. Moreover, the study sought to identify and assess independent variables, such as clinical and socio-demographic factors, lifestyle and the psychological effects of the COVID-19 pandemic that may be related to the development of NP among college students.

\section{Materials And Methods}




\section{Study design}

A cross-sectional study was conducted in the form of an online questionnaire survey posted at the Emek Yezreel College in January 2021, during the third COVID-19 lockdown in Israel.

\section{Setting}

College students were invited to participate in the study. An online survey was circulated to students recruited through their electronic mail. Informed consent was obtained for experimentation with human subjects online. Participants recorded their responses online. Data were collected from January 7 to February 8, 2021.

\section{Ethics}

The survey was conducted in adherence to the Helsinki declaration and approved by the Departmental Research Ethics Committee, Emek Yezreel College (Approval Number YVC EMEK 2021-17). The survey conformed to the recommendations of the STROBE Statement.

\section{Participants}

Emek Yezreel College students were invited to participate in the study. The study had no exclusion criteria. We used the Qualtrics survey platform to administer all survey questionnaires in the form of an online survey circulated to students recruited through their electronic mail.

\section{Control Variable}

Sociodemographic and clinical questionnaire: The questionnaire was based on a previous research questionnaire for lower back pain that achieved reliable results in Hebrew [13]. We modified this questionnaire for NP. The questionnaire included sociodemographic variables such as gender, age, height, weight, and body mass index (BMI) (calculated as weight in kilograms divided by square height in meters); medications for NP taken in the last month (yes/ no); marital status, children (yes/ no), number of children; religiosity (secular, partly religious, religious); department of studies: health sciences, nursing, social sciences; degree being pursued (bachelor's degree, master's degree); year of studies (from first to fourth year).

Participants were also asked about their health habits: smoking and engaging in physical activity during their leisure time (yes/no). Physical activity was measured by hours per week: 1-2 hours, 2-3 hours, 3-4 hours. Participants were also asked to report their total average sitting hours per day, as determined by the sample item: "How many hours a day do you spend in a sitting position?". The average number of sitting hours was classified into four categories: less than 1 hour, 1-3 hours, 3-5 hours, over 5 hours. Participants were also asked to rate their stress related to their studies on a scale ranging from 1 (none) to 4 (a lot). Due to the distribution of this variable, it was dichotomized into "a lot" vs. "some".

\section{Outcome variables}


1. NP frequency: Participants were asked to mark the frequency with which they experienced NP at four time points, over their lifetime, during the last year, during the last six months, and current (third lockdown in Israel), on a four-point scale ranging from never/seldom to almost every day (on a scale 1-4). NP was defined in accordance with the Bone and Joint Decade 2000-2010 Task Force as an ache or discomfort in the anatomical region of the neck with or without radiation to the head, trunk, and upper limbs ${ }^{18}$. The neck is defined as the posterior neck region from the superior nuchal line to the spine of the scapula and the side region down to the superior border of the clavicle and the suprasternal notch [19].

2. Neck Disability Index (NDI) [20]: Participants answered the NDI questionnaire that included ten questions, each rated on a five-point scale ranging from 0 = "painless" to 5 = "worst pain imaginable." The maximum score was 50 . A higher score indicated a high disability rating (NDI), where Cronbach's alpha $a=.87$. The cut-off value of the NDI for detecting NP associated with disability was determined to be 15 , such that $0-14$ indicated no disability, while a score of 15 and up pointed to disability.

3. Perceived Stress Scale (PSS) [21]: Participants answered questions about their feelings and thoughts over the past month on a 4-point scale ( $1=$ never; 4 = often), with a high score reflecting a high level of stress. The questionnaire score is calculated by averaging the items. Cronbach's alpha was a $=.89$. The questionnaire includes eight positively worded items $[4,5,6,7,8,9,10,13]$ and six negatively worded items (items $1,2,3,11,12,14$ ). Higher scores indicate higher levels of perceived stress.

4. Pain intensity: The visual analogue scale (VAS)[22] is a 10-cm line, oriented vertically or horizontally, with one end representing "no pain" and the other end representing "pain as bad as it can be." Patients are asked to mark the place on the line corresponding to their current pain intensity. The VAS is the most frequently used pain measure because it is simple to use and has good validation [22].

\section{Data analysis}

The data were analysed with SPSS ver. 27 . The background characteristics and the study variables were described through means and standard deviations, as well as with frequencies and percentages as applicable. Internal consistencies were calculated for the study variables using Cronbach's a. Logistic regression models were calculated for all background characteristics and study variables, comparing them using the NDI cut-off of 15 [20]. Another logistic regression model was calculated to assess the odds of having at least a moderate neck-related disability. The independent variables included age, leisure time physical activity, sitting hours per day, study-related stress, PSS score, VAS score, and use of medications for NP during the past month. Significance level was set at 0.05 . As a result of the online data collection protocol, there were no missing data.

Sample size was calculated with G*Power 3.1 for a logistic regression with minimum OR of I.5, $a=.05$, and power of 0.80 [23]. According to the regression, the required sample size was 308 participants. The actual sample size of 295 participants resulted in power of 0.80 for $O R=1.6$.

\section{Results}


A total of 295 college students participated in the study. Table 1 shows the participants' demographic characteristics and academic characteristics during the pandemic. As can be seen from the table, most of the participants were female (85\%) and in their twenties $(M=27.7$ years, $S D=8.32$ years). Most were single $(67.5 \%)$ and had no children (73.9\%). Almost all were studying for a bachelor's degree $(94.9 \%)$ in the health sciences or nursing (76.6\%). Most did not smoke (81.4\%) and were not pregnant $(95.6 \%)$ at the time of the study. Table 1 also depicts participants' behavioural habits: $81.4 \%$ did not smoke and $45.8 \%$ of them engaged in some form of leisure time physical activity. Most (75.6\%) spent three hours a day sitting, and $50 \%$ spent more than five hours a day in a sitting position. About a third of the participating students took medication for NP during the last month.

According to the NDI, about a third of the sample $(n=105,35.6 \%)$ experienced at least moderate neckrelated disability, while about two-thirds $(n=190,64.4 \%)$ reported no significant neck-related disability. A comparison of the demographic, background, and health-related characteristics according to the NDI cutoff revealed no significant differences for all demographic and background characteristics: gender, age, family status, children and number of children, religiosity, department and year of studies (table 1). Further, no significant differences were found for several health-related characteristics: body mass index (BMI), smoking, physical activity, and weekly hours of physical activity. Sitting hours were associated with the NDI index, such that those who sat over three hours per day were at significantly higher risk for at least a moderate level of neck-related disability. Reporting at least moderate neck-related disability was related to higher odds of using medications for NP and experiencing higher levels of pain.

Participants reported the extent of their NP at four time points: lifetime, last year, last six months, and current. Their answered on a four-point scale, ranging from never/seldom to almost every day. The results showed that reported NP increased gradually and significantly, from a lifetime mean of $1.80(S D=1.01)$, to a mean of $2.57(S D=1.13)$ during the last year, to a mean of $2.73(S D=1.10)$ during the past six months, and to a current mean of $3.07(S D=1.11)\left(X^{2}(3)=316.72, p<.001\right)$ (Fig. 1). Currently reported NP (scale 1 to 4$)$ was highly associated with the VAS score (scale $1-10)(r=.68, p<.001)$, as well as with the NDI score $(r=.63, p<.001)$.

Participating students reported their stress using the PSS and a single question regarding their studyrelated stress (Table 2). The mean PSS was moderate, at about 2.5 on a scale from 1 to 4 . Reported stress was significantly associated with the NDI cut-off point, such that a higher level of stress was associated with greater odds of experiencing at least a moderate level of neck-related disability. Quite similarly, $59.3 \%$ of the students $(n=175)$ reported experiencing study-related stress. Stress exhibited a significant association with the NDI cut-off point, such that reporting a high level of study-related stress was associated with experiencing at least moderate neck-related disability. The two measures of stress were positively correlated $(r=.42, p<.001)$. 
Table 2

Distribution of stress for the total sample and by NDI $(N=295)$

\begin{tabular}{|c|c|c|c|c|c|c|}
\hline Variable & Range & Total & $\begin{array}{l}\text { NDI up to } \\
14 \\
(n=190)\end{array}$ & $\begin{array}{l}\text { NDI } 15 \text { and } \\
\text { higher } \\
(n=105)\end{array}$ & $O R(95 \% C l)$ & $p$ \\
\hline PSS, $M(S D)$ & $1-4$ & $\begin{array}{l}2.49 \\
(0.52)\end{array}$ & $2.34(0.47)$ & $2.76(0.50)$ & $\begin{array}{l}6.41 \\
(3.58, \\
11.48)\end{array}$ & $<.001$ \\
\hline $\begin{array}{l}\text { Study-related } \\
\text { stress, } \\
M(S D)\end{array}$ & $0-1$ & $\begin{array}{l}0.59 \\
(0.49)\end{array}$ & $0.47(0.50)$ & $0.82(0.39)$ & $\begin{array}{l}5.14 \\
(2.90,9.11)\end{array}$ & $<.001$ \\
\hline $\begin{array}{l}\text { Abbreviations } \\
\text { SD-Standard }\end{array}$ & eck Disa & ity Inde & -Perceived & ss Scale; M-I & & \\
\hline
\end{tabular}

A logistic regression model was used to assess the odds of reporting at least a moderate level of neckrelated disability. The independent variables were: age, physical activity (1-yes, 0 -no), number of hours sitting per day (1-over 3 hours, 0-up to 3 hours), study-related stress (1-high, 0-some), PSS score, VAS score (1-10), and use of medications for neck/shoulder pain during the past month (1-yes, 0-no) (Table 3 ). The logistic model was found to be significant $\left(\chi^{2}(7)=136.76, p<.001\right)$, explaining about $51 \%$ of the variance in NDI (Nagelkerke's $R^{2}=.513$ ). Sitting for more than three hours a day, a high level of studyrelated stress, higher PSS score, higher VAS score, and the use of medications for NP during the past month were associated with a greater risk of experiencing at least a moderate level of neck-related disability. 
Table 3

Logistic-regression model for NDI(cut-off) with background variables, physical-habits, stress, pain, and use of medications $(N=295)$

\begin{tabular}{|c|c|c|c|c|}
\hline & B & $\mathrm{SeB}$ & $O R(95 \% C l)$ & $p$ \\
\hline Age & 0.03 & 0.02 & $1.03(0.99,1.08)$ & .127 \\
\hline Physical activity (yes) & 0.35 & 0.33 & $1.42(0.74,2.71)$ & .293 \\
\hline Number of hours sitting per day (over 3 hours) & 1.52 & 0.46 & $4.57(1.86,11.20)$ & $<.001$ \\
\hline Study-related stress (high) & 0.77 & 0.38 & $2.15(1.03,4.51)$ & .042 \\
\hline PSS & 1.46 & 0.39 & $4.32(2.01,9.29)$ & $<.001$ \\
\hline VAS & 0.32 & 0.08 & $1.38(1.17,1.63)$ & $<.001$ \\
\hline Use of medications (yes) & 1.50 & 0.36 & $4.46(2.22,8.99)$ & $<.001$ \\
\hline \multicolumn{5}{|c|}{$\begin{array}{l}\text { Abbreviations: NDI-Neck Disability Index; VAS-Visual Analogue Scale; PSS-Perceived Stress Scale; OR: } \\
\text { Odds Ratio; Cl- Confidence Interval; P- P Value. }\end{array}$} \\
\hline \multicolumn{5}{|c|}{$\begin{array}{l}\text { Note. Age: continuous variable; physical activity: } 1 \text {-yes, } 0 \text {-no; sitting hours per day: } 1 \text {-over } 3 \text { hours, } 0 \text {-up } \\
\text { to } 3 \text { hours; study-related stress: } 1 \text {-high, } 0 \text {-some; VAS score: } 1 \text {-very low, } 10 \text {-very high); use of } \\
\text { medications for neck pain during the past month: } 1 \text {-yes, } 0 \text {-no. }\end{array}$} \\
\hline
\end{tabular}

\section{Discussion}

Lockdown is an effective way to prevent the spread of the COVID-19 virus [10].Yet the shift in academic studies from on-campus studies to online learning exerted negative effects on students. The COVID-19 lockdown had an adverse impact on NP among college students. The current study reported NP at four time points-lifetime, last year, last six months, and current-on a scale ranging from never/seldom to almost every day. The mean NP score increased gradually and significantly during the lockdown period. To the best of our knowledge, the current study is the first to assess NP at four time points among college students, particularly during the COVID-19 lockdown.

In addition, current reports of NP were highly associated with VAS scores, as well as with the scores on the NDI. Furthermore, the results indicate that about a third (35.6\%) of the college students reported at least a moderate degree of neck-related disability, while about two thirds of them (64.4\%) reported no significant disability.

Similar results emerged in recent a previous study [2], showing that $38.2 \%$ of female medical students experience at least moderate neck and shoulder pain, whereas about $60 \%$ of them reported mild or no symptoms. Even though the previous study [2] was conducted before the coronavirus pandemic, both studies exhibited same prevalence of moderate NP among students. Furthermore, the current study assessed NP severity and disability according to the NDI questionnaire, whereas the previous study [2] 
used one question from the Computer Vision Syndrome Questionnaire to assess the severity of neck and shoulder symptoms as mild, moderate or severe.

Other studies conducted before the COVID-19 pandemic also reported a prevalence of NP symptoms among academic students; NP was reported in $69 \%$ of students in Italy [25], $24 \%$ of medical students in Egypt [26], and $75.1 \%$ of students at the University of Jamaica [27], and $22.3 \%$ of students at a university in Thailand [28]. Since musculoskeletal pain in the neck and shoulder region depends on exposure to risk factors, such as stress, incorrect posture, number of hours sitting per day and electronic device use, the range is diverse.

Majumdar et al. [3] reported no adverse effects of the COVID-19 pandemic on NP. Another study conducted in Turkey found that during the three-month lockdown when workers stayed home, the number of complaints of NP in workers was lower compared to the pre-lockdown period [29]. The results of the current study are inconsistent with those of these two previous studies. The main reason for this difference lies in the fact that two different populations were examined. In both previous studies the participants were workers, while in the current study the participants were students.

A possible explanation for these findings is that most people who stayed at home spent less time working or did not work at all. Hence, their work hours and exposure to work-related physical stress may have been reduced [29].Indeed, the reduction in the rate of NP and musculoskeletal complaints may be attributed to a decrease in work hours [29]. In contrast, the study population in the current study consisted of students. During the lockdown, students spent more time sitting in front of screens and may therefore have been more exposed to physical stress and excessive use of electronic devices.

Furthermore, our results indicate that sitting more than three hours a day is associated with a higher risk of experiencing at least moderate neck-related disability. The lockdown increased students' reliance on electronic devices to connect online, resulting in increased screen time as well as sitting time. In a recent systematic review, working in sustained awkward positions was determined to be a risk factor associated with the development of NP [6].

During the COVID-19 lockdown, our student participants reported a moderate level of perceived stress. Moreover, about $60 \%$ of them reported study-related stress. Furthermore, higher levels of stress and studyrelated stress were also associated with higher odds for experiencing at least a moderate degree of neckrelated disability.

According to the $\mathrm{WHO}$, the pandemic crisis is generating stress throughout the population that can have a negative impact on physical and mental well-being [7]. Zhang et al [17] revealed that undergraduate students who are confined to their homes suffer from chronic stress. The literature about COVID-19 and psycho-emotional distress reports similar findings [30,31]. As in these studies, the current study found that psycho-emotional distress has a greater impact on students. According to Marelli et al [30], the lockdown in Italy had a significant impact both on sleep and on psycho-emotional well-being and this impact was greater among students than among administrative staff workers and greater among women 
than among men. Similarly, Li et al [31] found that $16.3 \%$ students at Wuhan universities and colleges had posttraumatic syndrome four months after the COVID-19 pandemic.

Our finding concerning the high prevalence of study-related stress among students provides further support for the results of a previous cross-sectional study, which demonstrated that NP is related to academic pressure [17].

Moreover, NP has been found to be associated with psychosocial and mental stress [3]; stress was found to be one of the strongest predictors of neck/shoulder pain [32]. Similarly, a high degree of mental stress is considered a risk factor for musculoskeletal disorders in the neck [33].

The results of the current study indicate that sitting more than three hours a day is associated with a greater risk of experiencing at least a moderate level of neck-related disability. The lockdown increased people's reliance on electronic devices to connect online, which in turn resulted in more time in front of the screen time as well as more time sitting.. A recent systematic review determined that working in sustained awkward positions is a risk factor associated with the development of NP [6].

One surprising result of the current study is that leisure time physical activity was not found to be associated with NP-related disability. This finding is inconsistent with previous studies, which found that physical activity reduced NP $[34,35]$ and served as a protective factor against NP [6]. However, most risk factors found for neck pain were related to psychosocial characteristics rather than to physical characteristics [6]. We believe that the psychosocial condition expressed by the stress students experienced during the pandemic period is the dominant cause of pain, rather than leisure time physical activity.

Our results also indicate that older age and smoking among students were not related to NP and disability during the lockdown period. The results of our study are consistent with those of a recent systematic review suggesting that age and smoking status, were not risk factors for developing NP [6].

Several potential sources of bias may be identified. First, potential participants may have chosen not to respond to the online questionnaire. Their characteristics compared to those of students who completed the questionnaire are unknown, constituting a limitation of the study. Second, the online protocol required that participants complete the questionnaire in full so as to avoid missing data. Third, social desirability may have biased the respondents' answers. For that reason, the data were collected anonymously. Fourth, the study design is cross-sectional, and retrospective questions were asked. Reporting may have been affected by current emotions or cognitions, constituting another limitation of the study. Finally, a larger sample size is needed in order to be able to generalize the conclusions of the current study.

\section{Conclusion}

Based on our findings, we can conclude that the transition from on-campus studies to online learning had negative effects on students. It significantly increased study-related stress and the development of NP. 
This study has several implications. Because the study was conducted empirically throughout the COVID19 pandemic, particularly during the lockdown period, it can help in assessing the implications of COVID19 on students. Moreover, the study characterized potential factors that help in identifying which students were more likely to develop NP during this pandemic period. These associations with specific factors (e.g., stress, study-related stress, number of hours sitting per day) can assist in locating potential risk groups among college students.

Academic decision-makers and health systems should note the finding that during the COVID-19 pandemic period college students were under a remarkable degree stress, particularly study-related stress, followed by an increase in complaints of NP. This finding should foster the development of intervention strategies and approaches, such as developing occupational, ergonomic and prevention programs in healthcare settings to reduce stress, study stress and musculoskeletal pain among students, while targeting relevant groups. Further research is needed to identify interventions that may reduce the high rates of injury and NP among students.

\section{Abbreviations}

NP-Neck Pain; NDI-Neck Disability Index; VAS- Visual Analogue Scale; PSS- Perceived Stress Scale; MMean; SD-Standard Deviation; OR: Odds Ratio; Cl- Confidence Interval

\section{Declarations}

Ethics approval and consent to participate: This study was conducted in accordance with the principles of the Declaration of Helsinki. Approval was granted by the Institutional Review Board of the Departmental Research Ethics Committee, Emek Yezreel College (YVC EMEK 2021-17).

Electronic informed consent was obtained from all individual participants included in the study. Participation in the study was voluntary and participants could withdraw their consent at any time without giving reasons or without consequences to themselves.

Consent for publication: Not applicable

Availability of data and materials: The datasets used and/or analysed during the current study are available from the corresponding author on reasonable request.

Competing interests: The authors declare that they have no competing interests

Funding: This study received no external funding.

Authors' contributions: Both authors designed and led the study, collected and analysed the data. Both authors were involved in the data interpretation, drafted the manuscript and revised it critically for important intellectual content. All authors have read and approved the final manuscript. 
Acknowledgements: Not applicable.

\section{References}

1. Al-Hadidi F, Bsisu I, AlRyalat SA, et al. Association between mobile phone use and neck pain in university students: A cross-sectional study using numeric rating scale for evaluation of neck pain. PLOS ONE 2019;14: e0217231.

2. Al Tawil L, Aldokhayel S, Zeitouni L, et al. Prevalence of self-reported computer vision syndrome symptoms and its associated factors among university students. Eur J Ophthalmol 2020;30:189195.

3. Majumdar P, Biswas A, Sahu S. COVID-19 pandemic and lockdown: cause of sleep disruption, depression, somatic pain, and increased screen exposure of office workers and students of India. Chronobiol Int 2020;37:1-10.

4. Weleslassie GG, Meles HG, Haile TG, et al. Burden of neck pain among medical students in Ethiopia. BMC Musculoskelet Disord 2020;21:14.

5. Pico-Espinosa O J, Côté P, Hogg-Johnson S, et al. Trajectories of Pain Intensity Over 1 Year in Adults With Disabling Subacute or Chronic Neck Pain. Clin J Pain 2019;35: 678-685.

6. Kim R, Wiest C, Clark K, et al. Identifying risk factors for first-episode neck pain: A systematic review. Musculoskelet Sci Pract 2018;33:77-83.

7. World Health Organization. Mental health and psychosocial considerations during the COVID-19 outbreak. 18 March 2020. Available from: https://apps.who.int/iris/bitstream/handle/10665/331490/WHO-2019-nCoV-MentalHealth-2020.1eng.pdf

8. Alsalameh A, Harisi M, Alduayji M, et al. Evaluating the relationship between smartphone addiction/overuse and musculoskeletal pain among medical students at Qassim University. J Family Med Prim Care 2019;8:2953.

9. Singh HK, Joshi A, Malepati RN et al. A Survey of E-Learning Methods in Nursing and Medical Education during COVID-19 Pandemic in India. Nurse Educ. Today 2021; 99:104796.

10. Fallon N, Brown C, Twiddy $\mathrm{H}$, et al. Adverse effects of COVID-19-related lockdown on pain, physical activity and psychological well-being in people with chronic pain. J. Pain 2020.

11. Alshagga MA, Nimer AR, Yan LP, et al. Prevalence and factors associated with neck, shoulder and low back pains among medical students in a Malaysian Medical College. BMC Res Notes 2013;6:244.

12. AlShayhan FA, Saadeddin M. Prevalence of low back pain among health sciences students. Eur $J$ Orthop Surg Traumatol 2018;28:165-170.

13. Ben-Ami N, Korn L. Associations between backache and stress among undergraduate students. J Am Coll Health 2020;68:61-67.

14. Madaan V, Chaudhari A. Prevalence and risk factor associated with musculoskeletal pain amongst students of MGM Dental College-a cross sectional survey. Int Dent J Stud Res. 2012;1. Available 
from: http://www.physics.csbsju.edu/cgibin/stats/contingency_form

15. Luchetti M, Lee JH, Aschwanden $\mathrm{D}$, et al. The trajectory of loneliness in response to COVID-19. Am Psychol 2020;75:897-908.

16. Son C, Hegde S, Smith A, et al. Effects of COVID-19 on college students' mental health in the United States: Interview survey study. J Med Internet Res 2020; 22.

17. Zhang Y, Deng G, Zhang Z, et al. A cross sectional study between the prevalence of chronic pain and academic pressure in adolescents in China (Shanghai). BMC Musculoskelet Disord 2015;16(1).

18. Guzman J, Hurwitz EL, Carroll LJ, et al. A new conceptual model of neck pain: Linking onset, course, and care: The Bone and Joint Decade 2000-2010 Task Force on Neck Pain and Its Associated Disorders. Spine J 2008;33(4 SUPPL.).

19. Misailidou V, Malliou P, Beneka A, et al. Assessment of patients with neck pain: a review of definitions, selection criteria, and measurement tools. J Chiropr Med 2010;9:49-59.

20. Kato S, Takeshita K, Matsudaira K, et al. Normative score and cut-off value of the Neck Disability Index. J Orthop Sci 2012;17:687-693.

21. Cohen S, Kamarck T, Mermelstein R. Perceived stress scale. Measuring stress: A guide for health and social scientists, 1994;10:1-2.

22. Price DD, McGrath PA, Rafii A, et al. The validation of visual analogue scales as ratio scale measures for chronic and experimental pain. Pain J1983;17:45-56.

23. Faul F, Erdfelder E, Lang AG, et al. G*Power 3: A flexible statistical power analysis program for the social, behavioral, and biomedical sciences. Behav Res Methods 2007;39:175-191.

24. Young IA, Dunning J, Butts R, et al. Reliability, construct validity, and responsiveness of the neck disability index and numeric pain rating scale in patients with mechanical neck pain without upper extremity symptoms. Physiother Theory Pract 2019;35:1328-1335.

25. Lorusso A, Bruno S, L'Abbate N. Disturbi muscolo-scheletrici in studenti universitari che utilizzano il computer. Med Lav. 2009;100:29-34. Available from: https://pubmed.ncbi.nlm.nih.gov/19263870/

26. Iqbal M, El-Massry A, Elagouz M et al. Computer Vision Syndrome Survey among the Medical Students in Sohag University Hospital, Egypt. Ophthalmic Res 2018;8:1-8.

27. Mowatt L, Gordon C, Santosh ABR., et al. Computer vision syndrome and ergonomic practices among undergraduate university students. Int J Clin Pract 2018;72:e13035.

28. Kanchanomai S, Janwantanakul P, Pensri P, et al. Prevalence of and factors associated with musculoskeletal symptoms in the spine attributed to computer use in undergraduate students. Work J 2012;43:497-506.

29. Toprak Celenay S, Karaaslan Y, Mete O, et al. Coronaphobia, musculoskeletal pain, and sleep quality in stay-at home and continued-working persons during the 3-month Covid-19 pandemic lockdown in Turkey. Chronobiol Int 2020;37:1778-1785.

30. Marelli S, Castelnuovo A, Somma A, et al. Impact of COVID-19 lockdown on sleep quality in university students and administration staff. J Neurol 2021;268:8-15. 
31. Li X, Fu P, Fan C, et al. COVID-19 Stress and Mental Health of Students in Locked-Down Colleges. J. Environ. Res. Public Health 2021;18:771.

32. Smedley J, Inskip H, Trevelyan F, et al. Risk factors for incident neck and shoulder pain in hospital nurses. Occup Environ Med 2003;60:864-869.

33. Smith DR, Mihashi M, Adachi Y, et al. A detailed analysis of musculoskeletal disorder risk factors among Japanese nurses. J Safety Res 2006;37:195-200.

34. Daher A, Carel RS, Tzipi K, et al. The effectiveness of an aerobic exercise training on patients with neck pain during a short- and long-term follow-up: a prospective double-blind randomized controlled trial. Clin Rehabil 2020;34:617-629.

35. Daher A, Dar G, Carel R. Effectiveness of combined aerobic exercise and neck-specific exercise compared to neck-specific exercise alone on work ability in neck pain patients: a secondary analysis of data from a randomized controlled trial. Int Arch Occup Environ Health 2021:1-12.

36. Reich A, Chatzigeorkidis E, Zeidler $C$, et al. Tailoring the cut-off values of the visual analogue scale and numeric rating scale in itch assessment. Acta Derm Vener 2017;97: 759-760.

\section{Table}

Due to technical limitations, table 1 PDF is only available as a download in the Supplemental Files section.

\section{Figures}




\section{Neck pain over time}

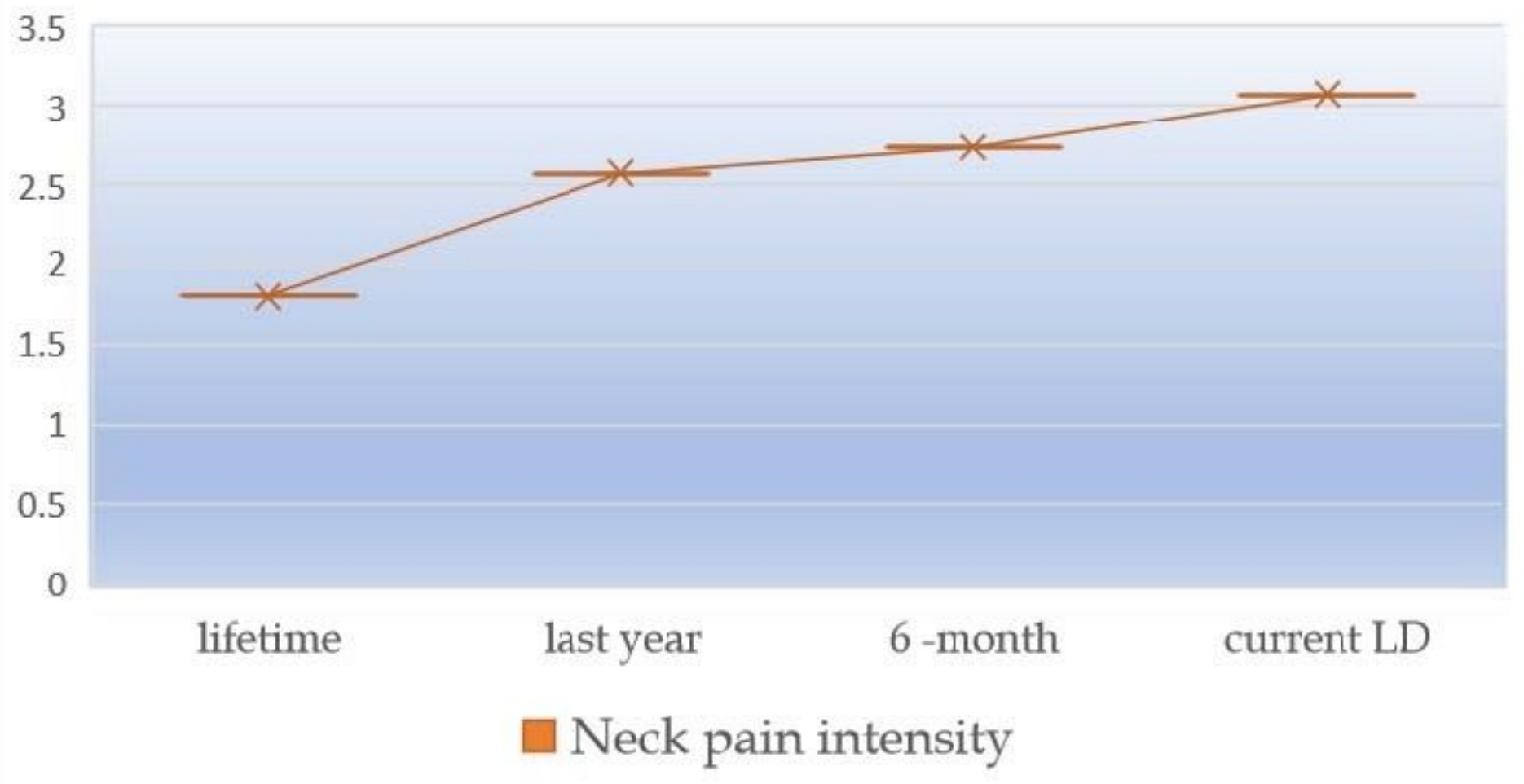

\section{Figure 1}

Neck-pain at four time-points: lifetime, last-year, 6-month, and current, ranging on a scale(1-4). Abbreviations: current LD: current lockdown

\section{Supplementary Files}

This is a list of supplementary files associated with this preprint. Click to download.

- filenumber2table1090721.pdf 